\title{
FINGER -VEIN TEMPLATE RECOGNITION SYSTEM USING CNNRESNET 18
}

\author{
Rahul Dev \\ Research Scholar, Department Of Electronics \& Communication Engg., \\ Galgotias University,Greater Noida, U.P., India \\ devmgm@rediffmail.com \\ Dr. Rohit Tripathi \\ Associate Professor, Department of Electronics \&Communication Engg., \\ Galgotias University,Greater Noida, U.P., India \\ rohittripathi30.iitd@gmail.com \\ Dr. Ruqaiya Khanam \\ Professor,Department of Computer Science Engineering, \\ Sharda University,Greater Noida, U.P., India \\ dr.kruqaiya@gmail.com
}

\begin{abstract}
Biometric Authentication Technology has been broadly utilized in area of retrieving information of peoples. As one of the most significant innovation of verification, finger vein recognition system is considered best because of its high security, solid precision and better accuracy. However, the system of finger vein recognition cannot be used widely because systems are based on complex image processing concept and they are not representative of feature vector. So here to solve this problem we have implemented CNN in developing Finger vein recognition system, Images are directly provided to the input of CNN for extracting its feature out with the goal that we can make validation by looking at the Euclidean distance between these vectors. We have developed a system with implementation of convolution neural network specifically resnet18 for the training image dataset and image retrieving process is done. Purpose of introducing deep learning in developing finger vein identification system is to get accurate more performance and speedy results. Results are computed on the basis Euclidean distance between features obtained from test image and features of trained images, the model designed has good robustness in illumination and rotation.
\end{abstract}

Keywords:Finger-vein recognition; Feature extraction; CNN, Euclidean distance, Resnet18

\section{Introduction}

Smart recognition of human personality for control and security is a worldwide issue of worry in our present reality. Money related misfortunes because of fraud can be extreme, and the respectability of security frameworks bargained. Henceforth, automatic confirmation frameworks for control have discovered application in criminal recognizable proof, self-governing distributing and computerized managing an account among others. Among the numerous authentication frameworks that have been developed and actualized, finger vein biometrics is developing as the fool proof technique for computerized individual distinguishing proof[1]. Finger vein is a remarkable physiological biometric for recognizing people in light of the physical qualities and traits of the vein designs in the human finger.

Palm print can be effectively frayed. Voice, marks can be effortlessly replicated or named. Face acknowledgment ends up troublesome on account of its event, for example, wearing cosmetics, glares, confront lifts, wearing caps or top. At exhibit there is need of cost effective, exact and dependable biometrics framework. Likewise, the state of the finger's surface (e.g., sweat, dryness) and skin mutilation can cause corrupted acknowledgment exactness. In spite of the fact that face acknowledgment hasfavorable circumstances in terms of client accommodation, its execution exceptionally relies upon facial articulations and light [2]. Iris acknowledgment is the most precise, in any case, the expense of catching gadget is high and it very well may be badly arranged since the client must adjust his iris to the camera [3]. To survive these issues, vein examples, like palm veins and hand veins have been contemplated. Vein acknowledgment utilizes vascular examples inside the human body. 
Finger vein detection system is proving one of the most accurate person's authentication system in these very low chances of forgery system. It is one of the reliable systems among other person's identifications system, in recent years, different methods have been created to address the security issue, however, there is still space for quick and effective biometric identification [4]. Biometric acknowledgment alludes to a programmed acknowledgment of individual properties procured by their anatomic/social qualities. A few kinds of biometric strategies have been introduced dependent on these anatomic features[5], for example, finger print, hand veins, finger veins, palm veins, foot vein, iris, stride, DNA acknowledgment, palates, voice acknowledgment, outward appearance, heartbeat, signature, non-verbal communication, and face shape. There are many biometric methods which are widely used such systems are typing patterns of persons, audio recognition, face recognition, signature recognition [6].Basically, these biometric system approaches can be partitioned into two classes 1) Extrinsic biometric features for example palm print recognition, iris recognition, fingerprint recognition 2) Intrinsic biometric features such as palm vein, finger vein and hand vein [7]. Just in case of visibility extrinsic biometric features are visible in comparison with intrinsic features,just for example during iris feature extraction our retina are exposed high intensity of light, features we get may be distorted that may affect, same in case of face recognition system[8],accuracy of identification may be distorted due to variation in present brightness, style of face and pose[9]. Advantages of finger vein identification over other biometric identifications are as follows: -

(1) Finger vein identification is non-contact biometric identification method, we don't have to touch anything with our fingers, for this reason it is accepted by users easily.

(2) Finger veins are internal characteristics of humans, so there will no chances of copying.

(3) All humans have 10 fingers we can use other finger in case any sudden change situation.

The conventional method of finger vein identification involves ROI separating, filtering, process of feature extraction; include noise removing and measuring distance matching [10]. Development of all these traditional methods generally takes a lot of time in pre-processing stage and different image sources are reasonable for various preparing techniques. So the proposed system for finger vein identification involves implementation of convolutional neural network (CNN)[11], it can take care of the issue of light change, scale change and picture rotation, prompting a magnificent performance on finger vein identification. In pattern recognition field finger vein detection is considered as complex part. Implementation CNN in this area makes this complex task into simple and easy, CNN is a variant of multilayer perceptron (MLP) that possesses build-in invariance [12]. Convolution neural network uses 2-dimnsional topology of the input image it provides way to make changes in input patterns, it was first introduced by Zhang[13], Basically applied multi-layer perceptron (MLP)and after that $\mathrm{Wu}$ and $\mathrm{Ye}[14]$ used probabilistic neural network technique and Liu made an examination dependent on Principal Component Analysis and ANFIS neuro fuzzy system and accomplished high precision of 99\%, however the execution time is 45.0s[15].Here in this paper we are implementing RESNET 18 convolutional neural network to achieve good accuracy at faster speed.

\section{Proposed Method}

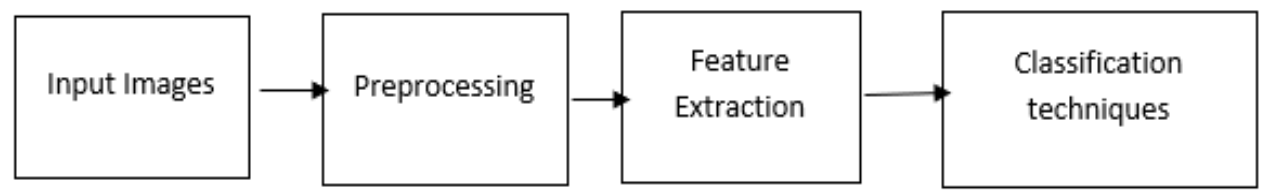

Fig. 1. Block Diagram of Proposed Method.

\subsection{Theory \& Principle}

Image preprocessing is one of the important tasks before proceed for the feature extraction, preprocessing is done to enhance the quality of image we have used histogram equalization method for image quality enhancement, Images from dataset SDU database. This databaseconsistsof 636 fingers, each with approx. 6 images, which was captured in one session [12]. These images are an 8-bit gray level BMP type with a resolution of $320 \times 240$ pixels. In our experiments, the images are preprocessed by our prior image preprocessing method these are obtained by placing finger in capturing device, from back of finger a infrared light is passed of approximate wavelength of 760-850 nm, radiation of light is absorbed by deoxyhemoglobin. Absorbing of light via hemoglobin let us display veins of finger's shadow. These veins patters are enhanced in preprocessing. 


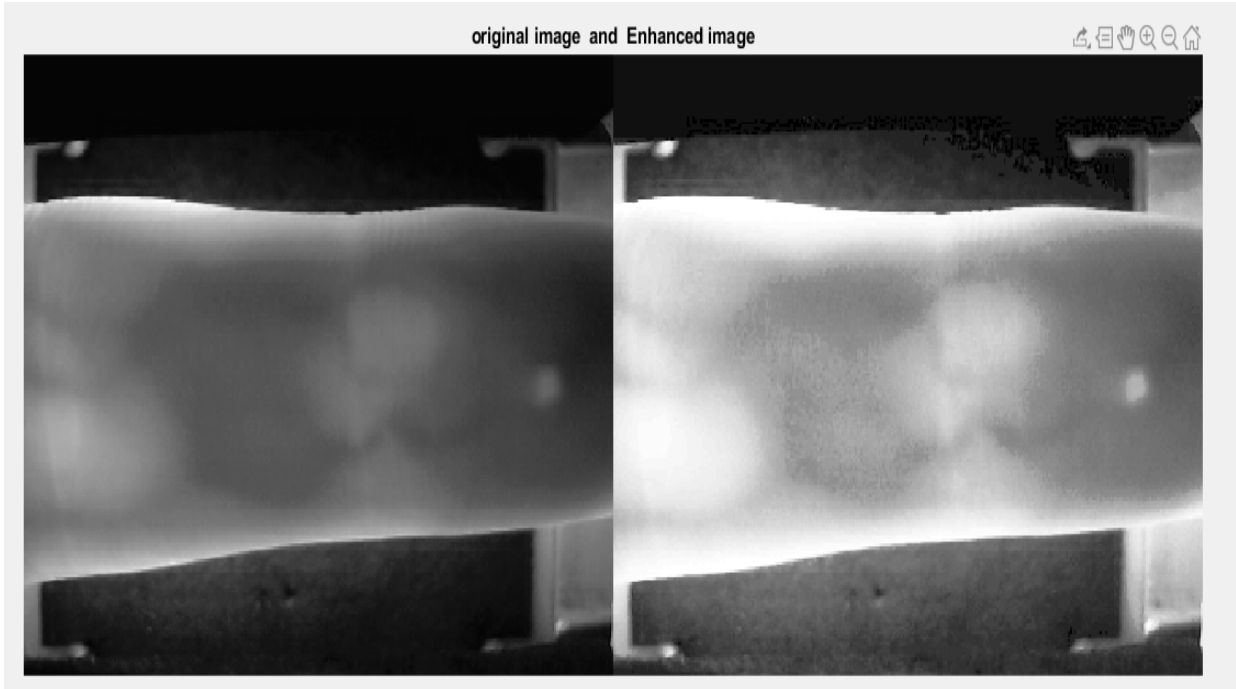

Fig. 2. Image enhanced by histogram equalization method.

Histogram equalization method involves adjustment of intensity values of provided image which ultimately increases contrast of the image,Histogramequalization is a specific category of histogram remapping methods [16]. As images of database have low visible intensity using this method there is transformation of intensity values so the histogram of the desired image is approximately gets matched. This method of image quality enhancement will help in further process of feature extraction.

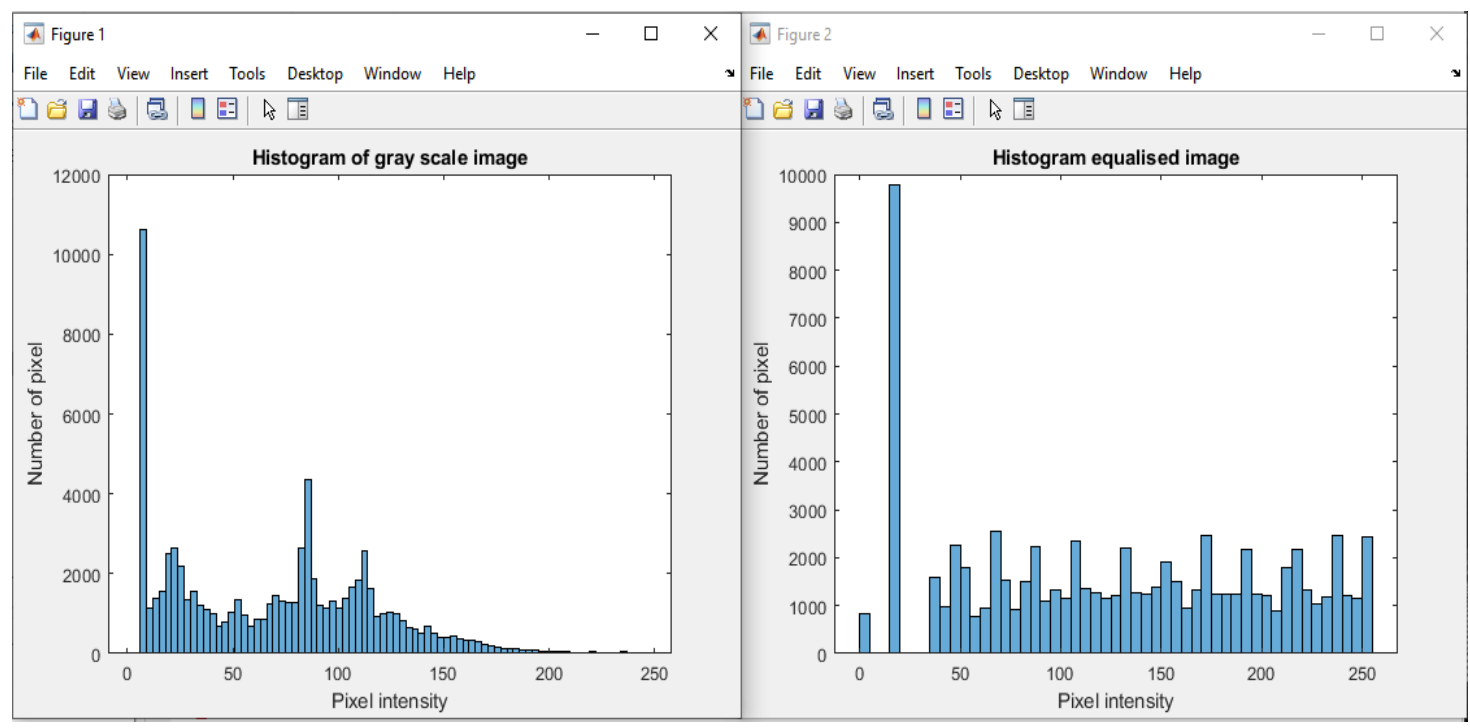

Fig. 3. Histogram of equalized image and gray scale image.

Enhancingof images obtained from histogram equalization as a pre-processingnext to implement morphological operations on set of images method to enhance images more so that feature extracted can be easily classified by different classification techniques.

\subsection{Morphological Operation}

Morphological operations are used to correct the non-uniform background illumination conversion of image into a binary image to make identification process easier, it is basically to visualize the clear veins from input images, it makes easy for the feature extraction process as it will help in findingthe area of veins and other statics for all other objects [17]. Preprocessing operation is done for conversionof colored and noisy image to enhanced image using different methods. Some results are displayed as:- 


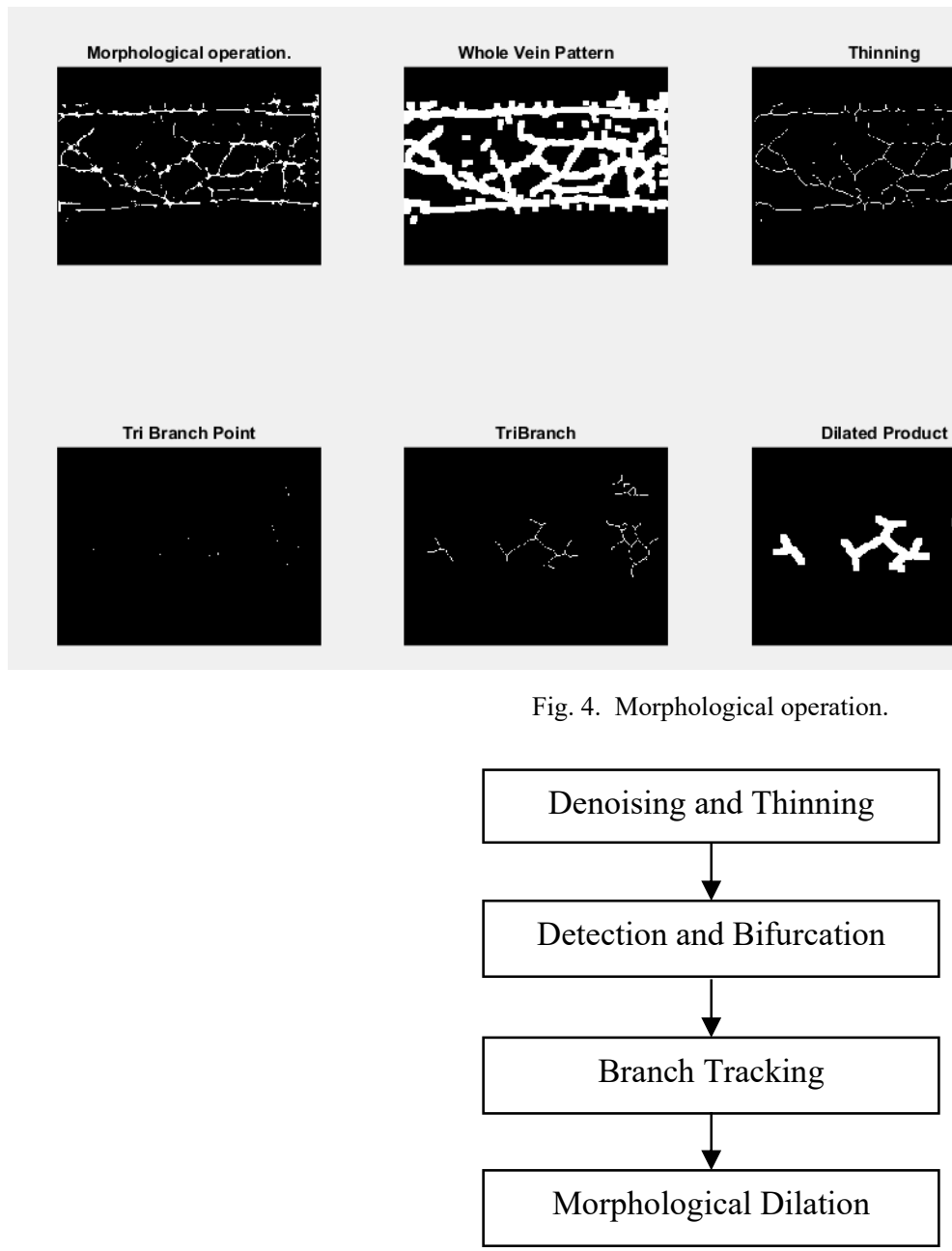

Fig. 5. Flowchart of Morphological operation

Morphological operations involve these operations as given below:-

\subsubsection{Denoising and Thinning}

The single-pixel wide vein arranges is removed from the vein design by the morphological diminishing task. The crossing point of the burr and vein branch can be erroneously observed as the bifurcation point.

\subsubsection{Detection of Bifurcation}

At one bifurcation point there are three connected vein branches. Expecting the present point what's more, its eight neighbor focuses are meant by $\mathrm{p}(\mathrm{x} ; \mathrm{y})$ and $\mathrm{P}=\mathrm{fp} 1 ; \mathrm{p} 2 ;:: ; \mathrm{p} 8 \mathrm{~g}$ individually. The point $\mathrm{p}(\mathrm{x} ; \mathrm{y})$ is viewed as a bifurcation, if Ns is equivalent to 6 , which is characterized as pursues.

\subsubsection{Branch Tracking}

$$
\mid \text { pi }+1-\text { pi } \mid \text {, where P9 = P1. }
$$

Three nonzero neighbor focuses can be recognized for one bifurcation point, and these neighbors are the underlying purposes of three vein branches.

\subsubsection{Dot product and Morphological dilation:}

Morphological expansion task is performed on the single-pixel wide tri-branch vein structure.

\subsection{Introduction to $C N N$}

A CNN or convolution neural network is a grouping of layers, and each layer changes one volume to another through differentiable functions. There are various types of layers in CNN.We should take a model by running a covnets on of picture of measurement $320 * 240$.

\subsubsection{Information Layer}

This layer holds the raw contribution of picture with width 320, height 240 . 


\subsubsection{Convolution Layer}

This layer computes the output volume by figuring dot product between all channels and picture fix.

\subsubsection{Actuation Function Layer}

This layer will apply component apply initiation function to the yield of convolution layer. Some basic actuation functions are RELU: $\max (0, \mathrm{x})$, Sigmoid: $1 /\left(1+\mathrm{e}^{\wedge}-\mathrm{x}\right)$, Tanh, Leaky RELU, and so on. .

\subsubsection{Pool Layer}

This layer is intermittently embedded in the covnets and its fundamental function is to decrease the size of volume which makes the calculation quick lessens memory and furthermore keeps from overfitting. Two normal sorts of pooling layers are max pooling and normal pooling.

CNN could be a gradable neural network that usually extracts features options by convolving input with a gaggle of kernel filters[18]. Then pooling of obtained feature is done and filtered resolute next layer. within the following, we are going to introduce $\mathrm{CNN}$ algorithm.

$$
x_{i}^{l} \in \mathbb{R}^{M i * M l}(2)
$$

Above given equation represents the lth layer of ith map, kernel filter of lth layer connected to the ith maps in the $(l-1)^{t h}$ layer and index map $M_{j}=\left\{i \mid i^{\text {th }}\right.$ set in the $(l-1)^{t h}$

$$
\mathrm{x}_{\mathrm{i}}^{\mathrm{l}}=\mathrm{f}\left(\sum_{\mathrm{i} \in \mathrm{M}_{\mathrm{j}}} \mathrm{x}_{\mathrm{i}}^{\mathrm{l}-1} * \mathrm{k}_{\mathrm{ij}}^{\mathrm{l}}+\mathrm{b}_{\mathrm{j}}^{\mathrm{l}}\right)(3)
$$

Above represents equation of the convolution operation. where $\mathrm{f}($.$) is the activation function which is known$ as ReLU non-linear $\mathrm{f}(\mathrm{z})=\max (0, \mathrm{z})$, where $b_{j}^{l}$ is bias

Pooling equation as-

$$
x_{i}^{l}=\operatorname{down}\left(x_{i}^{l-1}\right)
$$

Where sum-sampling function is down(.).

For multiclass classification an effective method is applied know as SoftMax regression, suppose for a given data you have $\mathrm{T}$ categories, training the training data for the each category are denoted as where $\mathrm{i}=\{1, \ldots \ldots \mathrm{N}\}$ $\left(x_{i}, y_{i}\right)$ with and,$x_{i}^{l} \in \mathbb{R}^{d} x_{i}^{l} \in \mathbb{R}$ are feature vector and labels apart

Cross entropy loss function given as-

$$
J(\theta)=-\frac{1}{N}\left[\sum_{i=1}^{N} \sum_{t=1}^{T} 1\left\{y_{i}=t\right\} \log \frac{e^{\theta_{t}^{T} x_{i}}}{\sum_{l=1}^{T} e^{\theta_{l}^{T} x_{i}}}\right](5)
$$

Here $\theta$ represents model parameters.

We are implementing residual network, the architecture of it is provided which specify the changes in layers at each step and depicts its performance and time taken at each step of execution. The system engineering incorporates five convolutional stages (see Table 1 for additional subtleties). The system is pre-prepared on the arrangement of pictures characterized by the Large-Scale Visual Recognition Challenge (ILSVRC )2015 test. The objective of this test is to recognize the features of finger veins and item classifications delineated in a photo. The complete number of classes is 1000 [19]. Despite the fact that the system is pre-prepared on scene and article pictures, it has illustrated, in primer analyses, to work far superior to a ResNet-18 pre-prepared on texture of pictures. The visual appearance of texture is surely increasingly like the visual appearance of the finger vein detection images considered right now. Despite this, the exhibition gotten by misusing the texture domain organize are a lot of more awful than the presentation got utilizing a scene-and article space one. All things considered, perceiving scenes and items is progressively entangled than perceiving surfaces, and in this way the system prepared to perceive scenes and items is progressively skilled of perceiving surprising irregular examples inside finger vein detection picture. Feature vector is obtained as linearized version of layers given output of the network. We explore different avenues regarding the utilization of two distinct layers of the system: the linearized yield of the fifth convolutional arrange (that is conv5 $\mathrm{x}$ ) and the yield of the normal pooling layer (that is avgpool). The size of the element vector is 25,088 (that is $7 \times 7 \times 512$ ) on account of the conv5_x layer and 512 on account of the avgpool layer. The size of the element vector influences the computational expense. 
Table1.ResNet-18 Architecture.

\begin{tabular}{|c|c|c|}
\hline Layer & Output Size & ResNet-18 \\
\hline conv1 & $112 \times 112 \times 64$ & $7 \times 7,64$, stride 2 \\
\hline conv2_x & $56 \times 56 \times 64$ & $\underline{3 \times 3 \text { max pool, stride } 2}$ \\
\hline conv3_x & $28 \times 28 \times 128$ & {$\left[\begin{array}{l}3 \times 3,64 \\
3 \times 3,64\end{array}\right] \times 2$} \\
\hline conv4_x & $14 \times 14 \times 256$ & {$\left[\begin{array}{l}3 \times 3,128 \\
3 \times 3,128\end{array}\right] \times 2$} \\
\hline conv5_x & $7 \times 7 \times 512$ & {$\left[\begin{array}{l}3 \times 3,256 \\
3 \times 3,256\end{array}\right] \times 2$} \\
\hline average pool & $1 \times 1 \times 512$ & {$\left[\begin{array}{l}3 \times 3,512 \\
3 \times 3,512\end{array}\right] \times 2$} \\
\hline fully connected & 1000 & $7 \times 7$ average pool \\
\hline softmax & 1000 & $512 \times 1000$ fully connections \\
\hline
\end{tabular}

3. Methodology toImplement in MATLAB

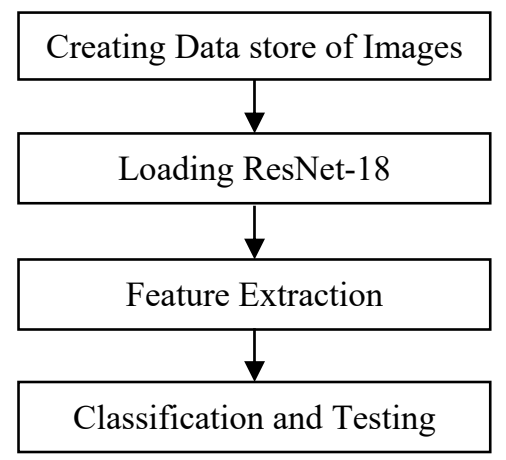

Fig. 6. Methodology flow chart

(1) Image datastore: -To train model wee first need to create datastore, to create data store we need to keep files in same directory, dataset contains 636 fingers images each with approximate 6 images.

(2) Division of datastore: -Next step is division of datastore into testing and training datastore to train models, we have taken $70 \%$ as training dataset and $30 \%$ as testing data.

(3) Loading ResNet 18:- In this step we have loaded the residual network RESNET 18 and analyzed the network it requires input size of image as $224 * 224 * 3$ where 3 represents the color channels. As we know images are not same size as we require for the network activation process, we have used augmented datastore where we can select desired input size of images. We have used activations on the pooling layers 5 represented as 'pool5', it is used pools the input vector overall spatial locations providing 512 features of each image.

(4) Classification and testing: -After training model on images we will test our images, we have created classifier and computed accuracy with testing data. After that we have tested classifier on query images.

\subsection{Classification techniques}

Classification can be performed on organized or unstructured information. Classification is where we classify information into a given number of classes. The principle objective of a classification issue is to recognize the classification/class to which information will fall under

\subsubsection{KNN (K- Nearest Neighbor)}

K-nearest neighbor normally known as KNN.It tends to be utilized for classification and problems based on regression. In addition, it is broadly utilized for classification [20]. KNN is calculation that fundamentally stores all the accessible individual instances of information and goes for classification of information on similitude 
measures. It arranges an information point on the premise that how its neighbors are characterized. K-means calculation is the most prevalent technique in AI, it depends on segment based bunching strategy.

First this calculation chooses explicit number of $\mathrm{k}$ bunch arbitrarily and next work on to pick each question the closest focus. In every cycle the normal of each bunch is shaped. This procedure iterated constantly till the target work become least or the maximum number of cycles finished.

\section{Steps involved in K-means-:}

(1) Chose irregular K case with arbitrary centroid.

(2) For each point allocate it to a bunch with closest centroid of the occasion.

(3) For each cluster recalculate the centroid dependent on the occasions present in that group.

(4) If the target work fulfilled, at that point stop; generally return to stage 2.

(5) The emphasis additionally stops if the aggregate of squared blunder is least, which is determined as follows where $\mathrm{Ck}$ is the $\mathrm{K}$ th cluster, mkis the centroid of the same cluster and $\mathrm{d}(\mathrm{x}, \mathrm{mk})$ is the distance between instance $\mathrm{x}$ and centroid $\mathrm{mk}$.

$\mathrm{E}=\sum_{x \in c k}^{n} d(x, m k)^{\wedge} 2$

Advantages: It is easy to implement it have capabilities to learn non-linear boundary, robust to noise in the input data.

Disadvantages: Inefficient since the entire training data is processed for every prediction. Time complexity is $\mathrm{O}(\mathrm{dMN} \log (\mathrm{k}))$ where $\mathrm{d}$ is the dimension of the data $\mathrm{M}$ the size of training data and $\mathrm{N}$ the size of test data.

\subsubsection{Linear discriminant}

Linear Discriminant Analysis or Normal Discriminant Analysis or Discriminant Function Analysis is a dimensionality decrease system which is ordinarily utilized for the managed order problem [21]. It is utilized for displaying contrasts in bunches for example isolating at least two classes. It is utilized to extend the highlights in higher measurement space into a lower measurement space. For instance, we have two classes and we have to isolate them proficiently. Classes can have various highlights. Utilizing just a solitary element to group them may bring about some covering as appeared in the beneath figure. Along these lines, we will continue expanding the quantity of highlights for legitimate characterization.

Assume we have two arrangements of information directs having a place toward two unique classes that we need to characterize. As appeared in the given 2D diagram, when the information focuses are plotted on the 2D plane, there's no straight line that can isolate the two classes of the information focuses totally. Subsequently, for this situation, LDA (Linear Discriminant Analysis) is utilized which decreases the 2D diagram into a 1D chart so as to expand the distinctness between the two classes. Two main limitations when the discriminative information are not in the means of classes and small sample size problem.

\section{Results \& Discussion}

After preprocessing we have proceeded with feature extraction process using network, Total number of extracted is 512 and classifying on basis of features extracted for all images, Testing process is done in samples ,results obtained from different classification techniques, In Testing process we need to test input query images , we have tested one query image on trained model, the accuracy we got as $100 \%$, we tried to input images in bunch or groups to check the accuracy, Accuracy we have received on different number of samples has bit variation in accuracy percentage this is because congestion of input data for trained model ,mismatch occur between features of input images and trained data features. Table of accuracy is given below and visualized by bar graphs.

Accuracy of the results is displayed as given below table:-

Table 2. Samples vs Accuracy.

\begin{tabular}{|c|c|}
\hline Number of Samples & Accuracy \\
\hline 50 samples & $97.5 \%$ \\
\hline 100 samples & 96.2 \\
\hline 120 samples & $95 \%$ \\
\hline 150 samples & $95.2 \%$ \\
\hline 200 samples & $93.5 \%$ \\
\hline
\end{tabular}

Above figure represents relation between sample vs accuracy as, we can observe that as the number of images in samples increases the accuracy decreases because mismatch occur between features of input images and trained data features. 


\subsection{ROC CNN}

ROC curve is a presentation estimation for classification issue at different limits settings. ROC is a likelihood curve and AUC tells about degree or proportion of distinguishableness. It tells how much model is fit for recognizing classes. Higher the AUC, better the model is at anticipating. In roc curve we generally focused on AUC (area of conversion) determines the goodness of the ROC curve.

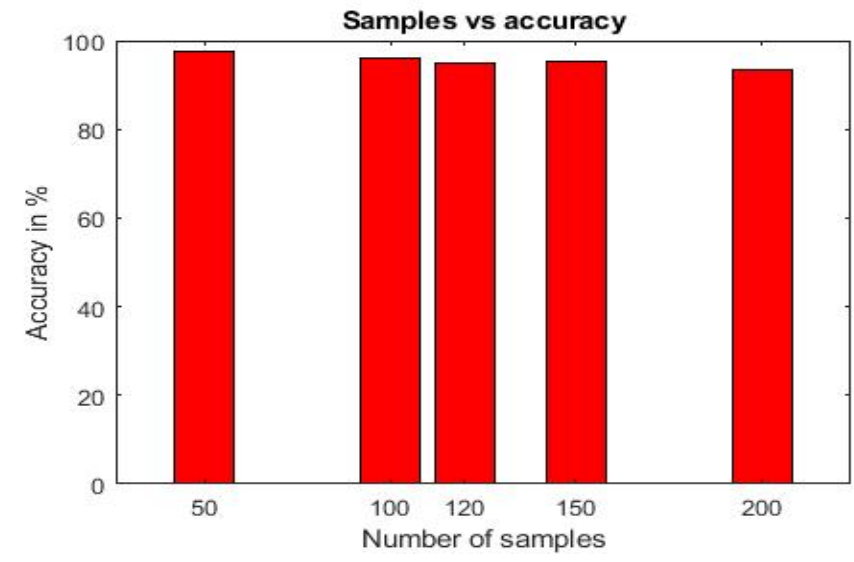

Fig. 7. Sample Vs accuracy of CNN technique.

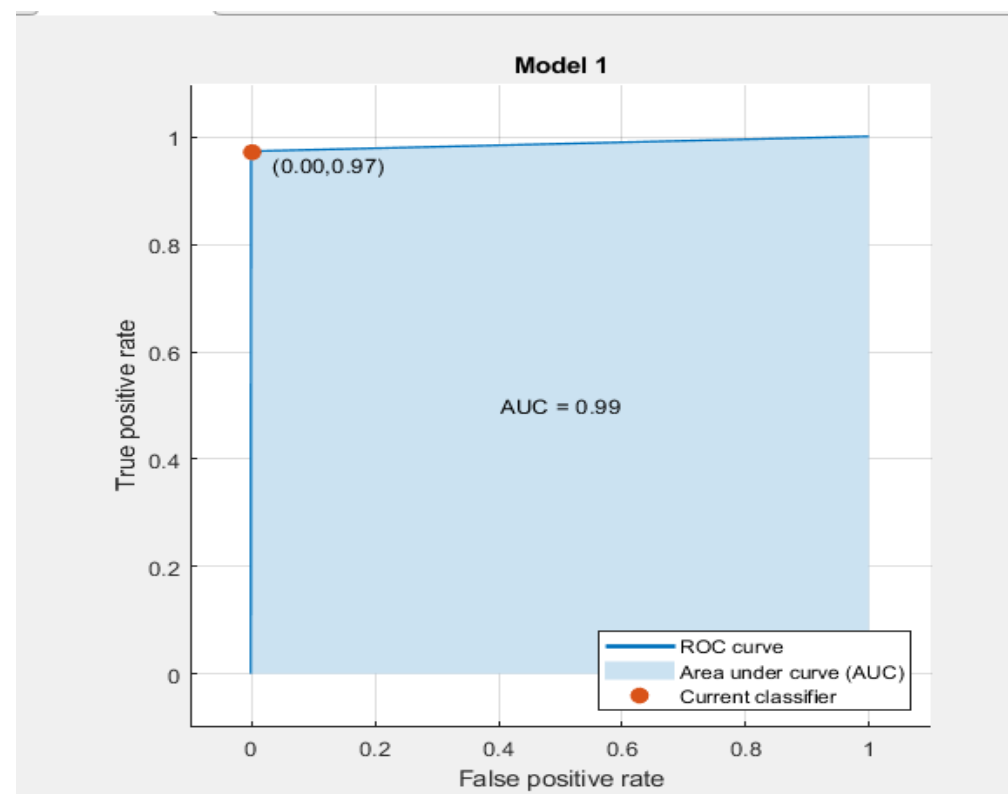

Fig. 8. ROC curve of CNN model.

\section{Comparison of Results}

Another finger vein identification framework is proposed in this paper. Especially, the features of finger vein which are extracted using CNN. Through the test, we can see that this strategy is exceptional both in speed and exactness. The Table 2 shows the accuracy examination between the CNN and other methods. It tends to be seen that there is no classification techniques better than CNN in terms of speed and accuracy for these complex problem.

Table 3 Comparison techniques on the basis of different techniques

\begin{tabular}{|c|c|}
\hline TECHNIQUES & ACCURACY \% \\
\hline KNN & $95.3 \%$ \\
\hline CNN & $99.9 \%$ \\
\hline LINEAR DISCRIMINANT & $94.5 \%$ \\
\hline
\end{tabular}




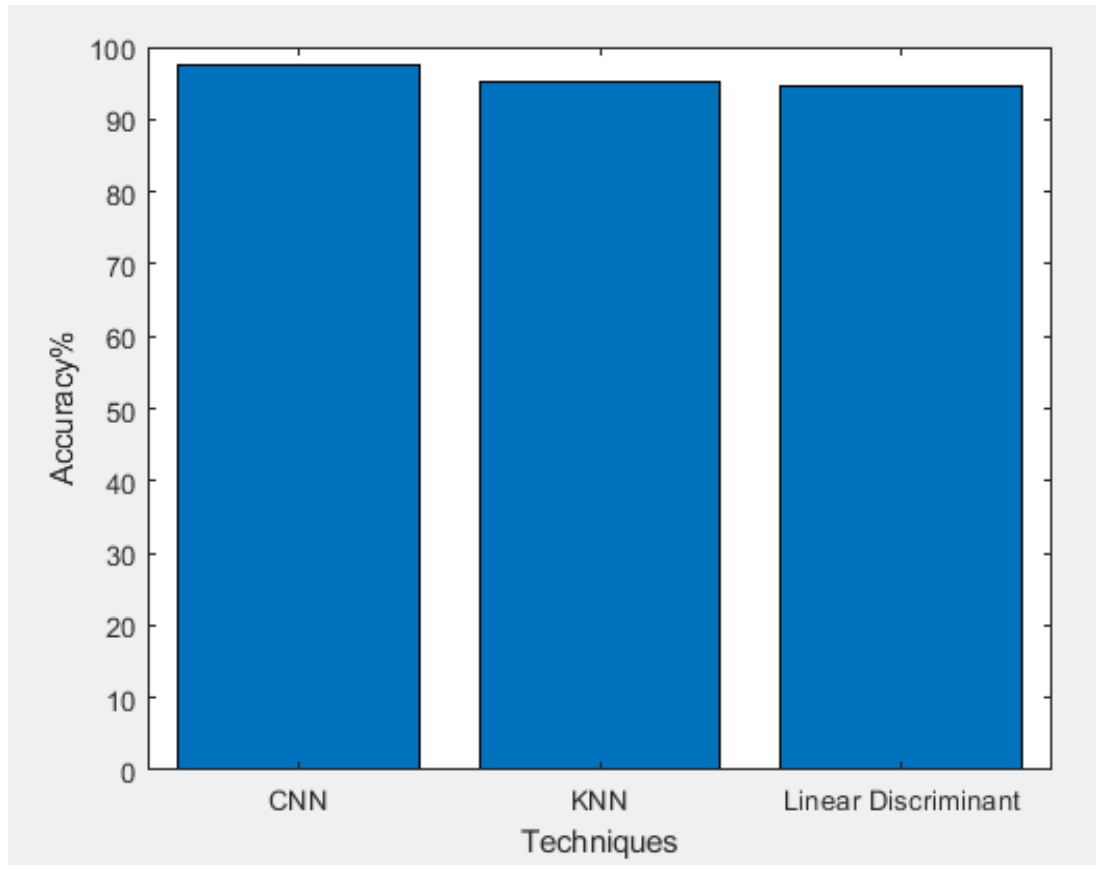

Fig.9.Comparison of Accuracy.

\section{Conclusion}

(1) Finger vein as a proof of validation, has a wide application possibility. In any case traditional method which is used have some complex process and as well as low speed.

(2) Our proposed system is prepared using CNN which has been proved to be more accurate and speedy system as in this system the ROI of images captured are directly input to the CNN to extract features then we compared the feature matching with the help of calculating Euclidean distance between two vectors.

(3) We concluded that CNN has highest accuracy among other two techniques, it has accuracy of $99.98 \%$ while KNN has $97.5 \%$ and Linear Discriminant 97.6\%.

\section{References}

[1] Duque, Jose, (2011) "Design and implementation of a finger vein identification system",November.

[2] S.Balakrishnan, "Biometric Authentication System Using Matlab," International Journal of Engineering and Technology., vol 7 ,No 19, (2018),pp 101-103.

[3] Gupta, Richa \& Sehgal, Priti, "A survey of attacks on iris biometric systems," International Journal of Biometrics,vol 8, No ,(2016),pp$145-178$.

[4] Duque, Jose"Design and implementation of a finger vein identification system", November (2011).

[5] X. HuafengQin, "Finger-vein verification based on the curvature in Radon space," Expert Systems with Applications, vol. 82, (2017), pp. 151-161.

[6] Wang, Ran \& Wang, Guoyou \& Chen, Zhong \& Zeng, Zhigang \& Wang, Yong, "A palm vein identification system based on Gabor wavelet features," Neural Computing \& Applications, vol 24,(2013),pp 161-168.

[7] Khalid, Syazana-Itqan \& Radzi, Feeza \& Mohd Saad, Norhashimah \& Abdul Hamid, Norihan \& Bin Mohd Saad, Wira Hidayat, "A Review of Finger-vein Biometrics Identification Approaches, "Indian Journal of Science and Technology,vol 9, No 32(2016).

[8] Kumar, Ashu \& Kaur, Amandeep \& Kumar, Munish, "Face Detection Techniques A Review,"Artificial Intelligence Review,(2018).

[9] Lu, Yu \& Wu, Shiqian \& Fang, Zhijun \& Xiong, Naixue \& Yoon, Sook \& Park, Dong., "Exploring finger vein based personal authentication for secure IOT," Future Generation Computer Systems. ,vol-77(2017).

[10] Liu, Zhi \& Song, Shangling, "An Embedded Real-Time Finger-Vein Recognition System for Mobile Devices,"Consumer Electronics, IEEE Transactions on,vol-58, No 2(2012),pp.522-527.

[11] Albawi, Saad \& Abed Mohammed, Tareq \& ALZAWI, Saad, "Understanding of a Convolutional Neural Network.," 2017.

[12] Yang, Lu \& Yang, Gongping \& Xi, Xiaoming \& Meng, Xianjing \& Zhang, Chunyun \& Yin, Yilong., "Tri-Branch Vein Structure Assisted Finger Vein Recognition,"IEEE Access,(2017),pp.1-1.

[13] Zhang, Zhongbo \& Ma, Siliang \& Han, Xiao, "Multiscale Feature Extraction of Finger-Vein Patterns Based on Curvelets and Local Interconnection Structure Neural Network," International Conference on Pattern Recognition, vol.4,(2006),pp. 145-148.

[14] Wu, Jian-Da \& Ye, Siou-Huan. "Driver identification using Finger-Vein Patterns with Radon Transform and Neural Network," Expert Systems with Applications,vol.36,(2009)pp. 5793-5799.

[15] Wu, Jian-Da \& Liu, Chiung-Tsiung, "Finger-vein pattern identification using principal component analysis and the neural network," Expert Systems with Applications, vol. 38, No 5(2011),pp. 5423-5427.

[16] Dorothy, R \& R M, Joany \& Rathish, Joseph \& Prabha, S \& Rajendran, Susai \& Joseph, " Image enhancement by Histogram equalization," International Journal of Nano Corrosion Science and Engineering, vol. 2, NO 4 (2015),pp. 21-30. 
[17] Yang, Lu \& Yang, Gongping \& Xi, Xiaoming \& Meng, Xianjing \& Zhang, Chunyun \& Yin, Yilong, "Tri-Branch Vein Structure Assisted Finger Vein Recognition," Digital Object Identifier IEEE Access ,vol.5,(2017),pp. 21020-21028.

[18] Albawi, Saad \& Abed Mohammed, Tareq \& ALZAWI, Saad, "Understanding of a Convolutional Neural Network.,"International Conference on Engineering and Technology (ICET), Antalya, (2017), pp. 1-6 .

[19] "ImageNet," [Online]. Available: http://www.image-net.org.

[20] Zhang, Zhongheng, "Introduction to machine learning: K-nearest neighbors," Annals of Translational Medicine, vol. 4, No 11 (2016),pp. 218-218

[21] Tharwat, Alaa \& Gaber, Tarek \& Ibrahim, Abdelhameed \& Hassanien, Aboul Ella, "Linear discriminant analysis: A detailed tutorial," Ai Communications.,vol.30,(2017),pp. 169-190.

[22] Meng, Gesi \& Fang, Peiyu \& Zhang, Bao, "Finger vein recognition based on convolutional neural network," International Conference on Electronic Information Technology and Computer Engineering, vol. 128,(2017) p. 5-13

[23] Leo Liberty, Carlile Lavor, Nelson Maculan, and Antonio Mucherino, "Euclidean Distance Geometry and Applications,", SIAM Review,vol. 56,(2012) 